\title{
Publisher Correction: Clinical efficacy and biomarker analysis of neoadjuvant atezolizumab in operable urothelial carcinoma in the ABACUS trial
}

Thomas Powles (D), Mark Kockx, Alejo Rodriguez-Vida, Ignacio Duran, Simon J. Crabb (D), Michiel S. Van Der Heijden, Bernadett Szabados, Albert Font Pous, Gwenaelle Gravis, Urbano Anido Herranz $\mathbb{B}$, Andrew Protheroe $\mathbb{D}$, Alain Ravaud, Denis Maillet, Maria Jose Mendez (10, Cristina Suarez, Mark Linch, Aaron Prendergast (1), Pieter-Jan van Dam (1), Diana Stanoeva, Sofie Daelemans, Sanjeev Mariathasan, Joy S. Tea B, Kelly Mousa, Romain Banchereau and Daniel Castellano

Correction to: Nature Medicine https://doi.org/10.1038/s41591-019-0628-7, published online 4 November 2019.

In the version of this article initially published, the labels in the key to Fig. 1 ( (red bars, Down in responders; blue bars, Up in responders) were incorrect. The correct labels are as follows: red bars, Up in responders; blue bars, Down in responders. Also, the histopathology images in Fig. 2a were incorrect. The correct images are now provided. The errors have been corrected in the HTML and PDF versions of the article.
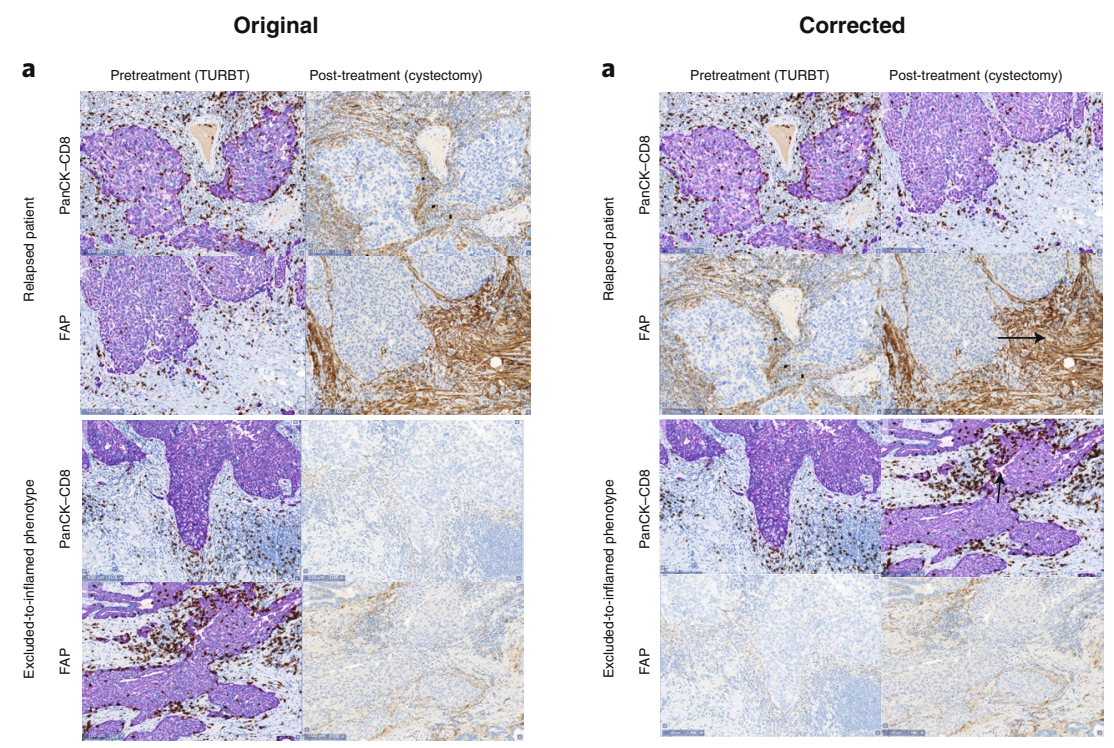

Fig. 2 | Original and Corrected.

Published online: 15 May 2020

https://doi.org/10.1038/s41591-020-0923-3

(c) The Author(s), under exclusive licence to Springer Nature America, Inc. 2020 